\title{
Poster Abstract: tCharge - A Fleet-Oriented Real-Time Charging Scheduling System for Electric Taxi Fleets
}

\author{
Guang Wang \\ Rutgers University \\ guang.wang@rutgers.edu
}

\author{
Fan Zhang \\ Shenzhen Institutes of Advanced \\ Technology, CAS \\ zhangfan@siat.ac.cn
}

\author{
Desheng Zhang \\ Rutgers University \\ desheng.zhang@cs.rutgers.edu
}

\begin{abstract}
With more and more advanced sensing and communication devices deployed on vehicles, ubiquitous vehicle location data (e.g., GPS) are available, which provide us great opportunities to enhance their mobility and energy performance (e.g., better scheduling decisions for operation or charging of taxis). In this paper, we design a real-time charging scheduling system called tCharge to address the charging problem of electric taxis with a fleet-oriented fashion. We leverage historical GPS data to estimate the travel time of electric taxis to different charging stations, and we utilize real-time GPS data of electric taxis to infer their waiting times at different charging stations. Then all these obtained information is fed to an online optimization for a fleet-oriented scheduling decision. We implement tCharge with real-world data from the Chinese city Shenzhen, including GPS data, taxi transaction data, road network data, and charging station data from more than 1,000 electric taxis and 28 charging stations. The results show our $t$ Charge outperforms existing methods by $82 \%$ of the queuing time reduction.
\end{abstract}

\section{CCS CONCEPTS}

- Information systems $\rightarrow$ Location based services; • Networks $\rightarrow$ Cyber-physical networks.

\section{KEYWORDS}

Electric taxi; fleet-oriented; charging scheduling, electric vehicle

\section{ACM Reference Format:}

Guang Wang, Fan Zhang, and Desheng Zhang. 2019. Poster Abstract: tCharge - A Fleet-Oriented Real-Time Charging Scheduling System for Electric Taxi Fleets. In The 17th ACM Conference on Embedded Networked Sensor Systems (SenSys '19), November 10-13, 2019, New York, NY, USA. ACM, New York, NY, USA, 2 pages. https://doi.org/10.1145/3356250.3361950

\section{INTRODUCTION}

As an important mode of urban transport, gasoline consumption, and emissions of gas taxis undoubtedly bring great challenges to the sustainable development of cities [3]. Compared to the conventional gas taxis, the burgeoning electric taxis (ETs) have great advantages. First, ETs behave smoother and make passengers feel

Permission to make digital or hard copies of all or part of this work for personal or classroom use is granted without fee provided that copies are not made or distributed for profit or commercial advantage and that copies bear this notice and the full citation on the first page. Copyrights for third-party components of this work must be

honored. For all other uses, contact the owner/author(s).

SenSys '19, November 10-13, 2019, New York, NY, USA

(C) 2019 Copyright held by the owner/author(s).

ACM ISBN 978-1-4503-6950-3/19/11 ..\$15.00

https://doi.org/10.1145/3356250.3361950 more comfortable. Second, ETs have absolutely no tailpipe emission and has the potential to overcome the foreseeable shortfall of fossil fuel worldwide [1]. Besides, ETs can also relieve urban noise pollution, which is a huge nuisance to many people. Considering these reasons, ETs are initially promoted in several large cities like New York City [2], Beijing and the Chinese city Shenzhen [3, 4]. The number of ETs in Shenzhen has increased from 50 in 2010 to 800 in 2015, and it is predicted that the number of ETs in Shenzhen will be more than 18000 by 2020 [4]. However, even though the obvious advantages, ETs have not been adopted nationwide due to the beleaguered charging problem. Long charging time shortens the overall operation time and leads anxiety to taxi drivers who make livings by them. In addition, unbalanced spatial distribution of charging stations and insufficient of fast charging points cause some ETs need to wait a very long time to have access to an available charging point. This may result in taxi drivers do not adopt ETs and make it worse for the taxi industry. Therefore, solving the charging problem is the key to promote the ETs at full blast.

However, it is extremely challenging to solve the charging problem of ET fleet. Different from private electric vehicles (PEVs), ETs cruise all day long to meet passengers' traveling demands and drivers' living needs. Due to the limited battery capacity, ETs need to be charged several times in a day, and each charge lasts for half to two hours. In addition, drivers usually choose the nearest charging stations to recharge their ETs. This heuristic-based charging behaviors also makes this situation worse, e.g., cause a unpredictable waiting time. In addition, ET drivers usually go to the charging stations in the lunch or dinner time when they can have meals both have the ETs charged. However, the limited charging stations make it hard to satisfy the intensive charging demands [3].

In this paper, we design a fleet-oriented real-time charging scheduling system called tCharge, which provides a promising solution to solve the sophisticated ET charging problem. In addition to status of a particular ET, we also utilize real-time status of the entire ET fleet, which provides more comprehensive information to infer various status of ETs and charging stations, e.g., the queuing time at charging stations and the potential future charging requests, which has not been considered by existing one-by-one scheduling [4].

\section{SYSTEM DESIGN}

\subsection{Data Description}

With more and more sensing and communication devices deployed on taxis, ubiquitous location data (e.g., GPS data) and transaction data are available for operation management and research. In this work, we are collaborating with Shenzhen Transportation Committee and have access to multi-source data, including GPS data, transaction data, charging station data, and road network data of 
this city. In this work, we utilize real-world GPS and transaction data collected from more than 1,000 ETs, combined with data of 28 charging stations to study ET charging patterns and evaluate our tCharge system.

\subsection{System Framework}

As shown in Figure 1, a three-layer data-driven system called tCharge for electric taxi fleet modeling and charging scheduling is designed in this work. The bottom layer is the multi-source data layer, which collects and stores data from the ubiquitous taxi infrastructure and lays the data foundation for charging modeling and scheduling. The middle layer is the charging activity modeling layer, which models the charging activities of ET fleets, including seeking and traveling activities to charging stations, queuing activity in stations for available charging points, and charging service activity at stations. Based on the charging modeling layer, we have many practical applications, e.g., charging station deployment and charging scheduling. In the top layer, i.e., the charging scheduling layer, we use the information obtained from the charging modeling layer to schedule ETs to the optimal charging stations, and the overall objective is to minimize the total charging time of all ETs and maximize the operating time of the fleet. After each scheduling decision, the status of charging stations will be recorded in the multi-source data layer, which will be used by modeling and scheduling layers again for a real-time iterative process.

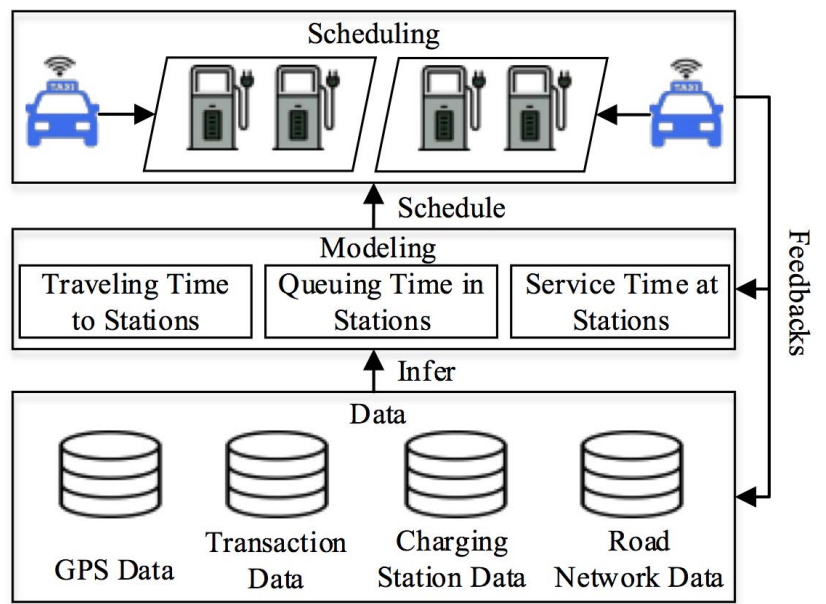

Figure 1: Overview of the tCharge.

\section{EVALUATION RESULTS}

We compare the performance of different scheduling methods using the metric Percentage of Reduced Queuing Time. Figure 2 and Figure 3 give the Percentage of Reduced Queuing Time in different hours for workdays and weekends. The negative numbers denote that the average queuing time using this method is longer than the Ground Truth. This is because too many ETs operate in urban business areas. If using Nearest Distance-Based Scheduling (NDBS) and Shortest Queuing Time-Based Scheduling (STBS), there are too many ETs will be scheduled to the same charging stations, leading to an increase of the queuing time. Our fleet-oriented tCharge also achieves better performance than the Individual-Oriented Optimal Scheduling (IOOS). During charging peak hours in one day, e.g., 3:00-6:00, 11:00-13:00, 16:00-18:00 [3], it will take more time for drivers to wait for an available charging point. The charging temporal clustering causes some existing scheduling methods having worse performance than Ground Truth.
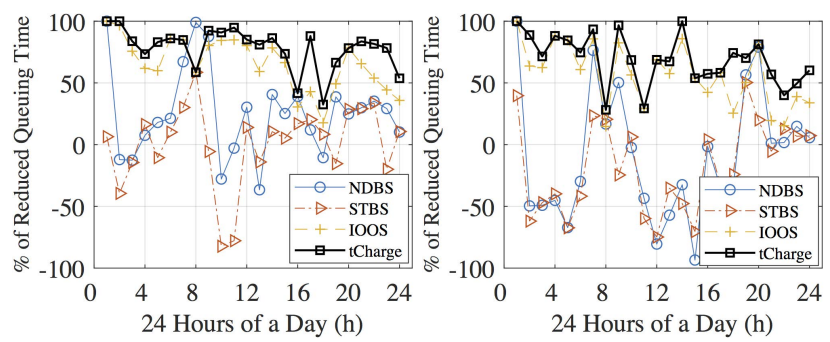

Figure 2: Queuing time re-Figure 3: Queuing time reduction in workdays. duction in weekends.

Since not all ET drivers will follow our scheduling, so we also evaluate the system performance under different driver following rates, and the results show our system still achieves good performance even only $50 \%$ drivers follow our scheduling decisions. Our tCharge system has the potential to help ET drivers to reduce their charging time, and it can also provide operators a better fleet management strategy.

\section{CONCLUSION}

In this paper, we design a fleet-oriented real-time charging scheduling system called tCharge based on historical and real-time multisource data, which aims to obtain an optimal charging scheduling strategy for the entire ET fleet, instead of individual vehicles. In the charging activity modeling layer, a vehicle-specific energy and time consumption model at road segment levels is designed based on the distances and the traffic conditions. Then this model is leveraged to infer the traveling time, queuing time, and service time of a particular ET based on the status of all relevant ETs in the fleet. Our system has the potential to increase drivers' profits due to the reduced charging time. Real-world data from Shenzhen city, including GPS data, taxi transaction data from more than 1,000 ETs, road network data and charging station data are leveraged to verify the performance of our tCharge. The results show tCharge effectively reduce the queuing time by $82 \%$.

\section{REFERENCES}

[1] Jacky Bourgeois, Stefan Foell, Gerd Kortuem, Blaine A. Price, Janet van der Linden, Eiman Y. Elbanhawy, and Christopher Rimmer. 2015. Harvesting green miles from my roof: an investigation into self-sufficient mobility with electric vehicles. In Proceedings of the 2015 ACM International foint Conference on Pervasive and Ubiquitous Computing, UbiComp 2015, Osaka, Japan, September 7-11, 2015. 10651076.

[2] NYC Taxi, Limousine Commission, et al. 2013. Take charge: A roadmap to electric New York City taxis.

[3] Guang Wang, Xiuyuan Chen, Fan Zhang, Yang Wang, and Desheng Zhang. 2019. Experience: Understanding Long-Term Evolving Patterns of Shared Electric Vehicle Networks. In Proceedings of the 25th annual international conference on Mobile computing and networking (MobiCom). ACM.

[4] Guang Wang, Wenzhong Li, Jun Zhang, Yingqiang Ge, Zuohui Fu, Fan Zhang, Yang Wang, and Desheng Zhang. 2019. sharedCharging: Data-Driven Shared Charging for Large-Scale Heterogeneous Electric Vehicle Fleets. Proceedings of the ACM on Interactive, Mobile, Wearable and Ubiquitous Technologies 3, 3 (2019), 108. 